\title{
The complete cd-index of Boolean lattices
}

\author{
Neil J.Y. Fan* \\ Department of Mathematics \\ Sichuan University \\ Chengdu, Sichuan, P.R. China \\ fan@scu.edu.cn
}

\author{
Liao He \\ Department of Mathematics \\ Sichuan University \\ Chengdu, Sichuan, P.R. China \\ scuhlj@126.com
}

Submitted: Mar 15, 2015; Accepted: May 24, 2015; Published: Jun 3, 2015

Mathematics Subject Classifications: 05A19, 05E15, 20F55

\begin{abstract}
Let $[u, v]$ be a Bruhat interval of a Coxeter group such that the Bruhat graph $B G(u, v)$ of $[u, v]$ is isomorphic to a Boolean lattice. In this paper, we provide a combinatorial explanation for the coefficients of the complete cd-index of $[u, v]$. Since in this case the complete cd-index and the cd-index of $[u, v]$ coincide, we also obtain a new combinatorial interpretation for the coefficients of the cd-index of Boolean lattices. To this end, we label an edge in $B G(u, v)$ by a pair of nonnegative integers and show that there is a one-to-one correspondence between such sequences of nonnegative integer pairs and Bruhat paths in $B G(u, v)$. Based on this labeling, we construct a flip $\mathcal{F}$ on the set of Bruhat paths in $B G(u, v)$, which is an involution that changes the ascent-descent sequence of a path. Then we show that the flip $\mathcal{F}$ is compatible with any given reflection order and also satisfies the flip condition for any cd-monomial $M$. Thus by results of Karu, the coefficient of $M$ enumerates certain Bruhat paths in $B G(u, v)$, and so can be interpreted as the number of certain sequences of nonnegative integer pairs. Moreover, we give two applications of the flip $\mathcal{F}$. We enumerate the number of cd-monomials in the complete cd-index of $[u, v]$ in terms of Entringer numbers, which are refined enumerations of Euler numbers. We also give a refined enumeration of the coefficient of $\mathbf{d}^{n}$ in terms of Poupard numbers, and so obtain new combinatorial interpretations for Poupard numbers and reduced tangent numbers.
\end{abstract}

Keywords: complete cd-index, cd-index, Boolean lattice, Bruhat graph

*Supported by the National Science Foundation of China (Grant No. 11401406) and the Research Fund for the Doctoral Program of Higher Education of China (Grant No. 20130181120103). 


\section{Introduction}

Let $W$ be a Coxeter group and $u, v \in W$ such that $u<v$ in the Bruhat order. The complete cd-index $\widetilde{\psi}_{u, v}(\mathbf{c}, \mathbf{d})$ of the interval $[u, v]$ is a nonhomogeneous polynomial in the noncommuting variables $\mathbf{c}$ and $\mathbf{d}$, which was introduced by Billera and Brenti [2] and conjectured to have nonnegative coefficients, see also Billera [1]. The complete cdindex $\widetilde{\psi}_{u, v}(\mathbf{c}, \mathbf{d})$ encodes compactly the ascent-descent sequences of the Bruhat paths in the Bruhat graph $B G(u, v)$ of $[u, v]$, and its combinatorial invariance is equivalent to the combinatorial invariance of the celebrated Kazhdan-Lusztig and $R$-polynomials, see $[2,12]$.

In [2], Billera and Brenti also showed that $\widetilde{\psi}_{u, v}(\mathbf{c}, \mathbf{d})$ is a generalization of the cd-index $\psi_{u, v}(\mathbf{c}, \mathbf{d})$ of $[u, v]$ in the sense that $\psi_{u, v}(\mathbf{c}, \mathbf{d})$ is the sum of the highest degree terms of $\widetilde{\psi}_{u, v}(\mathbf{c}, \mathbf{d})$. For the definition of the cd-index of an Eulerian poset, see, e.g., Stanley [16]. Purtill [15] gave a combinatorial interpretation for the $\mathbf{c d}$-index $\psi_{B_{n}}(\mathbf{c}, \mathbf{d})$ of the Boolean lattice $B_{n}$ by showing that $\psi_{B_{n}}(\mathbf{c}, \mathbf{d})$ is the sum of the $\mathbf{c d}$-variation monomials of augmented André permutations on $[n]:=\{1,2, \ldots, n\}$, and then derived a recursive formula for $\psi_{B_{n}}(\mathbf{c}, \mathbf{d})$. Besides, $\psi_{B_{n}}(\mathbf{c}, \mathbf{d})$ is also a refined enumeration of simsun permutations, which were first introduced by Simion and Sundaram [17, 18], see also Hetyei [9].

In this paper, we give a combinatorial interpretation for the coefficients of the complete cd-index of $[u, v]$, where $B G(u, v)$ is isomorphic to the Boolean lattice $B_{n}$. Since in this case all the edges in $B G(u, v)$ are covering relations, $\widetilde{\psi}_{u, v}(\mathbf{c}, \mathbf{d})$ has no lower degree terms. Hence we have

$$
\widetilde{\psi}_{u, v}(\mathbf{c}, \mathbf{d})=\psi_{u, v}(\mathbf{c}, \mathbf{d})=\psi_{B_{n}}(\mathbf{c}, \mathbf{d}) .
$$

Thus we also obtain a new combinatorial interpretation for the coefficients of the cd-index of the Boolean lattice $B_{n}$.

To this end, we label a directed edge in $B G(u, v)$, say $x \rightarrow y$, by a pair of nonnegative integers $(i, j)$, where $i$ (resp. $j$ ) is the number of edges $y \rightarrow z(y<z \leqslant v)$ such that the reflection $y^{-1} z$ is larger (resp. smaller) than the reflection $x^{-1} y$ in a given reflection order $\mathcal{O}$. Then we show that there is a one-to-one correspondence between the Bruhat paths in $B G(u, v)$ and the sequences of nonnegative integer pairs

$$
\left(\left(i_{1}, j_{1}\right),\left(i_{2}, j_{2}\right), \ldots,\left(i_{n}, j_{n}\right)\right),
$$

such that $i_{k}+j_{k}=n-k$ for $1 \leqslant k \leqslant n$.

Based on this labeling, we construct a flip $\mathcal{F}$ on the set of Bruhat paths in $B G(u, v)$, which is an involution that changes the ascent-descent sequence of a path. We show that the flip $\mathcal{F}$ is compatible with the reflection order $\mathcal{O}$ and also satisfies the flip condition for any cd-monomial $M$ defined by Karu [11]. Then the coefficient of $M$ enumerates certain Bruhat paths in $B G(u, v)$. Such paths are called valid paths and their corresponding sequences are called valid sequences. Therefore the coefficient of $M$ is the number of certain valid paths in $B G(u, v)$ or certain valid sequences of nonnegative integer pairs.

We give two applications of the flip $\mathcal{F}$. Let $E_{n}$ denote the Euler number, i.e., the number of up-down (or alternating) permutations on $[n]$. Denote by $E_{n}(k)$ the number of up-down permutations of length $n$ beginning with $k(1 \leqslant k \leqslant n)$. Clearly, we have 
$E_{n}=\sum_{k=1}^{n} E_{n}(k)$. Purtill [15] showed that the number of cd-monomials in $\psi_{B_{n}}(\mathbf{c}, \mathbf{d})$ is the Euler number $E_{n}$, that is,

$$
\psi_{B_{n}}(1,1)=\widetilde{\psi}_{u, v}(1,1)=E_{n} .
$$

We give a refined enumeration of $\widetilde{\psi}_{u, v}(1,1)$ in terms of the Entringer numbers $E_{n}(k)$. To be more specific, we show that the number of valid sequences of length $n$ beginning with $(n-k, k-1)$ or the number of valid paths in $B G(u, v)$ with first edge labeled by $(n-k, k-1)$ is equal to $E_{n}(k)$.

As the second application, we give a refined enumeration of the coefficient of $\mathbf{d}^{n}$ in $\widetilde{\psi}_{u, v}(\mathbf{c}, \mathbf{d})$ in terms of the Poupard numbers $P_{n}(k)(1 \leqslant k \leqslant 2 n+1)$, where $B G(u, v)$ is isomorphic to $B_{2 n+1}$. The paths in $B G(u, v)$ corresponding to the monomial $\mathbf{d}^{n}$ are called alternating paths. We show that the number of alternating paths with first edge labeled by $(2 n-k+1, k-1)$ is equal to the Poupard number $P_{n}(k)$. Since by [8], the reduced tangent number $t_{n}$ satisfies $t_{n}=\sum_{k=1}^{2 n+1} P_{n}(k)$, we deduce that the coefficient of $\mathbf{d}^{n}$ is the reduced tangent number $t_{n}$. Therefore we obtain new combinatorial interpretations for the Poupard numbers and reduced tangent numbers.

The organization of this paper is as follows. In Section 2, we give some basic notation and definitions on Coxeter groups and the complete cd-index. We also recall some results of Karu. In Section 3, we first construct a flip $\mathcal{F}$ on $[u, v]$ and show that this flip is compatible with the given reflection order and satisfies the flip condition. Then we provide a combinatorial interpretation for the coefficient of $\widetilde{\psi}_{u, v}(\mathbf{c}, \mathbf{d})$. In Section 4 , We give a refined enumeration of the number of cd-monomials in $\widetilde{\psi}_{u, v}(\mathbf{c}, \mathbf{d})$. Finally, in Section 5, we give a refined enumeration of the coefficient of $\mathbf{d}^{n}$ in $\widetilde{\psi}_{u, v}(\mathbf{c}, \mathbf{d})$.

\section{Preliminary}

Let $(W, S)$ be a Coxeter system, and let $T=\left\{w s w^{-1} \mid s \in S, w \in W\right\}$ be the set of reflections, see, e.g., Humphreys [10]. We use $\ell(w)$ to denote the length of $w \in W$. For $u, v \in W$, we say that $u \leqslant v$ in the Bruhat order if there exists a sequence of reflections $t_{1}, t_{2}, \ldots, t_{r}$ in $T$ such that (i) $v=u t_{1} t_{2} \cdots t_{r}$ and (ii) $\ell\left(u t_{1} \cdots t_{i-1}\right)<\ell\left(u t_{1} \cdots t_{i}\right)$ for $1 \leqslant i \leqslant r$. We say that $u$ is covered by $v$, if $u<v$ and $\ell(v)=\ell(u)+1$. Let $[u, v]=\{w \in$ $W \mid u \leqslant w \leqslant v\}$ be the interval formed by $u$ and $v$ in the Bruhat order. The atoms of $[u, v]$ are the elements $w \in[u, v]$ such that $w$ covers $u$.

The Bruhat graph $B G(W)$ of the Coxeter group $W$ is a directed graph whose vertices are the elements of $W$ and there is a directed edge from $u$ to $v$, denoted by $u \rightarrow v$, if $v=u t$ for some reflection $t \in T$ and $\ell(u)<\ell(v)$. The interval $[u, v]$ forms a subgraph $B G(u, v)$ of the Bruaht graph of $W$. A Bruhat path of length $n$ from $u$ to $v$ in $B G(u, v)$ is a sequence

$$
x=\left(u=x_{0} \rightarrow x_{1} \rightarrow \cdots \rightarrow x_{n-1} \rightarrow x_{n}=v\right)
$$

such that $t_{i}=x_{i-1}^{-1} x_{i} \in T$ for $1 \leqslant i \leqslant n$. We call $\left(t_{1}, t_{2}, \ldots, t_{n}\right)$ the reflection sequence of $x$, and call the first edge $u \rightarrow x_{1}$ of $x$ a first edge of the interval $[u, v]$. Let $B_{k}(u, v)$ denote the set of Bruhat paths of length $k$ from $u$ to $v$, and let $B(u, v)=\bigcup_{k} B_{k}(u, v)$. 
Recall that a reflection order $(\mathcal{O}, \prec)$ is a total order defined on the set of reflections, see Dyer [4]. The reverse of the order $\mathcal{O}$, denoted by $\overline{\mathcal{O}}$, is also a reflection order. Throughout this paper, we shall always use a given reflection order $(\mathcal{O}, \prec)$. We say that the path $x$ in (1) is increasing if $t_{1} \prec t_{2} \prec \cdots \prec t_{n}$, and decreasing if $t_{1} \succ t_{2} \succ \cdots \succ t_{n}$. Dyer [4] showed that each Bruhat interval $[u, v]$ is shellable. That is, there is a unique increasing (resp. decreasing) path of length $n$, say $x$ (resp. $y$ ), and the reflection sequence of $x$ (resp. $y$ ) is the lexicographically smallest (resp. largest) among all the reflection sequences of paths in $B_{n}(u, v)$.

The following result is due to Dyer [5].

Theorem 1. Let $x=\left(u \rightarrow x_{1} \rightarrow \cdots \rightarrow x_{n-1} \rightarrow v\right)$ be an increasing path in $B_{n}(u, v)$, and $y=\left(u \rightarrow y_{1} \rightarrow \cdots \rightarrow y_{m-1} \rightarrow v\right)$ be a decreasing path in $B_{m}(u, v)$. Then we have

$$
u^{-1} x_{1} \prec u^{-1} y_{1} \text { and } y_{m-1}^{-1} v \prec x_{n-1}^{-1} v .
$$

The ascent-descent sequence of the Bruhat path $x$ is a monomial in the noncommuting variables $\mathbf{a}$ and $\mathbf{b}$ defined by

$$
w(x)=w_{1} w_{2} \cdots w_{n-1},
$$

where

$$
w_{i}= \begin{cases}\mathbf{a}, & \text { if } t_{i} \prec t_{i+1} \\ \mathbf{b}, & \text { if } t_{i} \succ t_{i+1}\end{cases}
$$

The ab-index $\widetilde{\phi}_{u, v}(\mathbf{a}, \mathbf{b})$ of the interval $[u, v]$ is the polynomial obtained by summing the ascent-descent sequences of all the Bruhat paths from $u$ to $v$ :

$$
\widetilde{\phi}_{u, v}(\mathbf{a}, \mathbf{b})=\sum_{x \in B(u, v)} w(x) .
$$

The complete $\mathbf{c d}$-index $\widetilde{\psi}_{u, v}(\mathbf{c}, \mathbf{d})$ of the interval $[u, v]$ is obtained by a change of variable in the ab-index $\widetilde{\phi}_{u, v}(\mathbf{a}, \mathbf{b})$ of $[u, v]$. Let $\mathbf{c}=\mathbf{a}+\mathbf{b}$ and $\mathbf{d}=\mathbf{a b}+\mathbf{b a}$. Billera and Brenti [2] showed that $\widetilde{\phi}_{u, v}(\mathbf{a}, \mathbf{b})$ can be expressed in terms of $\mathbf{c}$ and $\mathbf{d}$ :

$$
\widetilde{\psi}_{u, v}(\mathbf{c}, \mathbf{d})=\widetilde{\psi}_{u, v}(\mathbf{a}+\mathbf{b}, \mathbf{a b}+\mathbf{b a})=\widetilde{\phi}_{u, v}(\mathbf{a}, \mathbf{b}) .
$$

It can be shown that $\widetilde{\psi}_{u, v}(\mathbf{c}, \mathbf{d})$ does not depend on the reflection order.

Now we proceed to recall some definitions and results in [11].

For an ab-monomial $M$, denote by $\bar{M}$ the ab-monomial obtained by exchanging $\mathbf{a}$ and $\mathbf{b}$ in $M$. This operator is an involution on the noncommutative ring $\mathbb{Z}\langle\mathbf{a}, \mathbf{b}\rangle$.

Definition 2. A flip $F=F_{u, v}$ on $[u, v]$ is defined to be an involution

$$
F_{u, v}: B(u, v) \rightarrow B(u, v),
$$

such that $w(F(x))=\overline{w(x)}$ for any path $x \in B(u, v)$. 
Fix a flip for every interval in the Bruhat graph of $W$. Let $1 \leqslant m \leqslant n$ and

$$
x=\left(u=x_{0} \rightarrow x_{1} \rightarrow \cdots \rightarrow x_{m} \rightarrow x_{m+1} \rightarrow \cdots \rightarrow x_{n} \rightarrow x_{n+1}=v\right)
$$

be a path in $B(u, v)$. After applying the flip $F_{x_{m}, v}$ to $x$, we obtain

$$
y=\left(u=x_{0} \rightarrow x_{1} \rightarrow \cdots \rightarrow x_{m} \rightarrow y_{m+1} \rightarrow \cdots \rightarrow y_{n} \rightarrow y_{n+1}=v\right) .
$$

If $w(x)=\beta_{1} \cdots \beta_{m} \cdots \beta_{n}$, then $w(y)=\beta_{1} \cdots \beta_{m-1} \alpha_{m} \bar{\beta}_{m+1} \cdots \bar{\beta}_{n}$, where $\alpha_{m}$ can be either a or b. Define

$$
\begin{aligned}
& s_{m, \mathbf{a}}(x)= \begin{cases}1, & \text { if } \beta_{m}=\mathbf{a} \\
0, & \text { otherwise }\end{cases} \\
& s_{m, \mathbf{b}}(x)= \begin{cases}1, & \text { if } \beta_{m}=\mathbf{b}, \alpha_{m}=\mathbf{a} ; \\
-1, & \text { if } \beta_{m}=\mathbf{a}, \alpha_{m}=\mathbf{b} ; \\
0, & \text { otherwise }\end{cases}
\end{aligned}
$$

Let the variables $\mathbf{a}, \mathbf{b}, \mathbf{c}$ have degree 1 , and let the variable $\mathbf{d}$ have degree 2 . Given a cd-monomial $M(\mathbf{c}, \mathbf{d})$ of degree $n$, we can obtain a unique ab-monomial $M(\mathbf{a}, \mathbf{b a})$ of degree $n$ by substituting $\mathbf{a}$ for $\mathbf{c}$ and ba for $\mathbf{d}$ in $M(\mathbf{c}, \mathbf{d})$. Clearly, this is a one-to-one correspondence between $\mathbf{c d - m o n o m i a l s ~ a n d ~} \mathbf{a b}$-monomials in which every $\mathbf{b}$ is followed by an $\mathbf{a}$.

Definition 3. Let $M(\mathbf{c}, \mathbf{d})$ be a $\mathbf{c d}$-monomial such that $M(\mathbf{a}, \mathbf{b a})=\gamma_{1} \gamma_{2} \cdots \gamma_{n}$. Define

$$
s_{M}(x)=\prod_{m=1}^{n} s_{m, \gamma_{m}}(x) .
$$

Note that $s_{m, \gamma_{m}}(x)$, and hence $s_{M}(x)$, depend on both the reflection order and the given flip. Denote by $\bar{s}_{m, \gamma_{m}}(x)$ the value of $s_{m, \gamma_{m}}(x)$ by using the reverse reflection order $\overline{\mathcal{O}}$, and let $\bar{s}_{M}(x)=\prod_{m=1}^{n} \bar{s}_{m, \gamma_{m}}(x)$.

Definition 4. A flip $F$ is said to be compatible with the reflection order $\mathcal{O}$ if

$$
s_{M}(x)=\bar{s}_{M}(F(x))
$$

for any interval $[u, v]$, any path $x \in B(u, v)$ and any cd-monomial $M$.

Theorem 5. Assume that the flip $F$ is compatible with the reflection order $\mathcal{O}$. For any cd-monomial $M$ of degree $n$, the coefficient of $M$ in $\widetilde{\psi}_{u, v}(\mathbf{c}, \mathbf{d})$ is equal to

$$
\sum_{x \in B_{n+1}(u, v)} s_{M}(x) .
$$


If -1 does not appear in the above sum, then the coefficient of $M$ is clearly nonnegative. Therefore Karu [11] introduced the following flip condition.

Definition 6. The flip condition holds for the interval $[u, v]$ and monomial $M$ if for every path $x \in B(u, v)$ the following is satisfied. If $s_{m, \gamma_{m}}(x)=-1$ for some $m$, then there exists $k>m$ such that $s_{k, \gamma_{k}}(x)=0$.

Definition 7. Let $M(\mathbf{c}, \mathbf{d})$ be a cd-monomial of degree $n$ with $M(\mathbf{a}, \mathbf{b a})=\gamma_{1} \gamma_{2} \cdots \gamma_{n}$. Define

$$
T_{M}(u, v)=\left\{x \in B_{n+1}(u, v) \mid s_{m, \gamma_{m}}(x)=1, \text { for all } 1 \leqslant m \leqslant n\right\} .
$$

From Theorem 5 we have

Corollary 8. If the flip condition holds for the interval $[u, v]$ and monomial $M$, then the coefficient of $M$ in $\widetilde{\psi}_{u, v}(\mathbf{c}, \mathbf{d})$ is equal to $\left|T_{M}(u, v)\right|$ and hence is nonnegative.

In [11], Karu proved that when $M$ contains at most one $\mathbf{d}$, that is, $M=\mathbf{c}^{i}$ or $M=$ $\mathbf{c}^{i} \mathbf{d c}^{j}(i, j \geqslant 0)$, the flip condition holds by Theorem 1 . Then the coefficient of $M$ is nonnegative by Corollary 8. Recently, the authors showed that when $M=\mathbf{d c}^{i} \mathbf{d} \mathbf{c}^{j}(i, j \geqslant$ $0)$, the coefficient of $M$ is also nonnegative, see [7].

\section{Combinatorial interpretation of $\widetilde{\psi}_{u, v}(\mathrm{c}, \mathrm{d})$}

In this section, we first give a labeling scheme for the edges in $B G(u, v)$ and then construct a flip $\mathcal{F}$ on the set of paths in $B(u, v)$ based on the labels of the paths. By using the flip $\mathcal{F}$, we provide a combinatorial interpretation for the coefficients of $\widetilde{\psi}_{u, v}(\mathbf{c}, \mathbf{d})$.

Let $[u, v]$ be a Bruhat interval such that $B G(u, v)$ is isomorphic to the Boolean lattice $B_{n}$. In the following, we shall always refer to $[u, v]$ as such an interval if there is no further notification. Note that every edge in $B G(u, v)$ is a covering relation. In fact, if there is an edge $u_{1} \rightarrow u_{2}$ in $B G(u, v)$ such that $u_{2}=u_{1} t$ for some $t \in T$ and $\ell\left(u_{2}\right)-\ell\left(u_{1}\right)>1$, then we must have $\ell\left(u_{2}\right)-\ell\left(u_{1}\right)=2 k+1$ for some $k \geqslant 1$. This implies $B G\left(u_{1}, u_{2}\right)$ would not be isomorphic to a Boolean lattice. Hence $B(u, v)=B_{n}(u, v)$, i.e., all the paths from $u$ to $v$ have length $n$.

Suppose that

$$
x=\left(u=x_{0} \rightarrow x_{1} \rightarrow x_{2} \rightarrow \cdots \rightarrow x_{n}=v\right)
$$

is a path in $B(u, v)$. For $1 \leqslant k \leqslant n$, label the edge $x_{k-1} \rightarrow x_{k}$ by an integer pair $\left(i_{k}, j_{k}\right)$, where $i_{k}$ (resp. $j_{k}$ ) is the number of first edges $x_{k} \rightarrow z$ in the interval $\left[x_{k}, v\right]$ such that $x_{k}^{-1} z \succ x_{k-1}^{-1} x_{k}$ (resp. $\left.x_{k}^{-1} z \prec x_{k-1}^{-1} x_{k}\right)$. Call the sequence $\left(\left(i_{1}, j_{1}\right), \ldots,\left(i_{n}, j_{n}\right)\right)$ the label sequence of the path $x$.

Proposition 9. Suppose that the atoms of $[u, v]$ are $u_{1}, u_{2}, \ldots, u_{n}$. Then the set of labels of the first edges $u \rightarrow u_{k}(1 \leqslant k \leqslant n)$ of $[u, v]$ is

$$
\{(i, j) \mid i+j=n-1,0 \leqslant i \leqslant n-1\} .
$$

Moreover, the label of $u \rightarrow u_{r}$ is lexicographically smaller than the label of $u \rightarrow u_{k}$ if and only if $u^{-1} u_{r} \succ u^{-1} u_{k}$. 
Proof. We make induction on $n$. By Theorem 1, it is easy to see that the proposition holds for $n=2$. Now assume that $n>2$. Since $[u, v]$ is shellable, there is a unique increasing (resp. decreasing) path $x$ (resp. $y$ ), and the reflection sequence of $x$ (resp. $y$ ) is the lexicographically smallest (resp. largest). Without loss of generality, we can assume

$$
x=\left(u \rightarrow u_{1} \rightarrow x_{2} \rightarrow \cdots \rightarrow x_{n}=v\right)
$$

and

$$
y=\left(u \rightarrow u_{n} \rightarrow y_{2} \rightarrow \cdots \rightarrow y_{n}=v\right) .
$$

So the reflection $u^{-1} u_{1}$ (resp. $u^{-1} u_{n}$ ) is the minimum (resp. maximum) among all the $n$ first edges of $[u, v]$. Moreover, since $x$ is increasing, $u^{-1} u_{1} \prec u_{1}^{-1} x_{2}$ and $u_{1}^{-1} x_{2}$ is the minimum among all the $n-1$ first edges of $\left[u_{1}, v\right]$. Similarly, since $y$ is decreasing, $u^{-1} u_{n} \succ u_{n}^{-1} y_{2}$ and $u_{n}^{-1} y_{2}$ is the maximum among all the $n-1$ first edges in $\left[u_{n}, v\right]$. Therefore, the label of the edge $u \rightarrow u_{1}$ is $(n-1,0)$ and the label of the edge $u \rightarrow u_{n}$ is $(0, n-1)$.

Notice that $u$ and the atoms $u_{2}, u_{3}, \ldots, u_{n-1}$ determine a Boolean lattice $B_{n-2}$. By induction, the set of labels of the first edges $u \rightarrow u_{k}(2 \leqslant k \leqslant n-1)$ in the Boolean lattice $B_{n-2}$ is

$$
\{(s, r) \mid s+r=n-3,0 \leqslant s \leqslant n-3\} .
$$

Since $B G(u, v)$ is a Boolean lattice, for each atom $u_{k}(2 \leqslant k \leqslant n-1)$, there exists $z_{k} \in[u, v]$ such that $u_{k} \rightarrow z_{k}$ and $u_{1} \rightarrow z_{k}$. Since $\ell\left(z_{k}\right)-\ell(u)=2$, by [3, Lemma 2.7.3], the interval $\left[u, z_{k}\right]$ has exactly two paths. Since $u^{-1} u_{1} \prec u_{1}^{-1} z_{k}$ and $\left[u, z_{k}\right]$ is shellable, we have $u^{-1} u_{k} \succ u_{k}^{-1} z_{k}$. Similarly, there exists $w_{k} \in[u, v]$ such that $u_{k} \rightarrow w_{k}$ and $u_{n} \rightarrow w_{k}$. Since $u^{-1} u_{n} \succ u_{n}^{-1} w_{k}$, we see that $u^{-1} u_{k} \prec u_{k}^{-1} w_{k}$. That is to say, if the edge $u \rightarrow u_{k}$ has label $(s, r)$ in $B_{n-2}$, then its label would be $(s+1, r+1)$ in $B_{n}$. Therefore, in the Boolean lattice $B_{n}$, the set of labels of the first edges is

$$
\{(i, j) \mid i+j=n-1,0 \leqslant i \leqslant n-1\} .
$$

It is clear that $u^{-1} u_{1}$ (resp. $u^{-1} u_{n}$ ) is the minimum (resp. maximum) among all the first edges of $[u, v]$ in the reflection order $\mathcal{O}$, and the label of $u \rightarrow u_{1}$ (resp. $u \rightarrow u_{n}$ ) is the largest (resp. smallest) in the lexicographic order. By induction, for $2 \leqslant k, r \leqslant n-1$, the label of $u \rightarrow u_{k}$ is lexicographically smaller than the label of $u \rightarrow u_{r}$ if and only if $u^{-1} u_{k} \succ u^{-1} u_{r}$ in $B_{n-2}$. Consequently, for $1 \leqslant k, r \leqslant n$, the label of $u \rightarrow u_{k}$ is lexicographically smaller than the label of $u \rightarrow u_{r}$ if and only if $u^{-1} u_{k} \succ u^{-1} u_{r}$ in $B_{n}$. This completes the proof.

Remark 10. According to Proposition 9, if we arrange the labels of the first edges $u \rightarrow$ $u_{1}, \ldots, u \rightarrow u_{n}$ of $[u, v]$ decreasingly in the lexicographic order, then the corresponding reflections $u^{-1} u_{1}, \ldots, u^{-1} u_{n}$ are arranged increasingly in the reflection order. Thus, without loss of generality, we can require the edge $u \rightarrow u_{k}$ to have label $(n-k, k-1)$ for $1 \leqslant k \leqslant n$. 
Corollary 11. There is a bijection between the Bruhat paths in $B(u, v)$ and sequences of nonnegative integer pairs $\left(\left(i_{1}, j_{1}\right), \ldots,\left(i_{n}, j_{n}\right)\right)$ such that $i_{k}+j_{k}=n-k$ for $1 \leqslant k \leqslant n$. In other words, a label sequence determines a unique path in $B(u, v)$ and vice versa.

Proposition 12. Let $u_{k-1} \rightarrow u_{k} \rightarrow u_{k+1}$ be two adjacent edges in $B(u, v)$ such that the edge $u_{k-1} \rightarrow u_{k}$ has label $\left(i_{k}, j_{k}\right)$ and the edge $u_{k} \rightarrow u_{k+1}$ has label $\left(i_{k+1}, j_{k+1}\right)$. Then

(1) $i_{k}>i_{k+1}$ if and only if $u_{k-1}^{-1} u_{k} \prec u_{k}^{-1} u_{k+1}$.

(2) $j_{k}>j_{k+1}$ if and only if $u_{k-1}^{-1} u_{k} \succ u_{k}^{-1} u_{k+1}$.

Proof. (1) Since there are $i_{k}$ edges among the first edges of $\left[u_{k}, v\right]$ which are larger than $u_{k-1}^{-1} u_{k}$, by Remark 10, we see that the labels of these $i_{k}$ edges are $(i, j)$ such that $0 \leqslant$ $i \leqslant i_{k}-1$. It follows that $i_{k}>i_{k+1}$ if and only if $u_{k-1}^{-1} u_{k} \prec u_{k}^{-1} u_{k+1}$. (2) Notice that $i_{k}+j_{k}=i_{k+1}+j_{k+1}+1$, then $i_{k}>i_{k+1}$ if and only if $j_{k} \leqslant j_{k+1}$. Thus $j_{k}>j_{k+1}$ if and only if $u_{k-1}^{-1} u_{k} \succ u_{k}^{-1} u_{k+1}$.

Now we can define a flip on $B(u, v)$ according to the labels of the edges.

Definition 13. Let

$$
x=\left(u=x_{0} \rightarrow x_{1} \rightarrow x_{2} \rightarrow \cdots \rightarrow x_{n}=v\right)
$$

be a path in $B(u, v)$ with label sequence $\left(\left(i_{1}, j_{1}\right),\left(i_{2}, j_{2}\right), \ldots,\left(i_{n}, j_{n}\right)\right)$. Define

$$
\mathcal{F}: B(u, v) \rightarrow B(u, v)
$$

as follows.

(1) If $\min \left\{i_{1}, j_{1}\right\} \leqslant \min \left\{i_{2}, j_{2}\right\}$, then let

$$
\mathcal{F}(x)=\left(u=x_{0} \rightarrow y_{1} \rightarrow y_{2} \rightarrow \cdots \rightarrow y_{n}=v\right)
$$

such that the label sequence of $\mathcal{F}(x)$ is $\left(\left(j_{1}, i_{1}\right),\left(j_{2}, i_{2}\right), \ldots,\left(j_{n}, i_{n}\right)\right)$.

(2) If $\min \left\{i_{1}, j_{1}\right\}>\min \left\{i_{2}, j_{2}\right\}$, then let

$$
\mathcal{F}(x)=\left(u=x_{0} \rightarrow x_{1} \rightarrow y_{2} \rightarrow \cdots \rightarrow y_{n}=v\right)
$$

such that the label sequence of $\mathcal{F}(x)$ is $\left(\left(i_{1}, j_{1}\right),\left(j_{2}, i_{2}\right), \ldots,\left(j_{n}, i_{n}\right)\right)$.

By Proposition 12 , it is easy to see that $\mathcal{F}$ is a flip on $B(u, v)$. For example, let $[u, v]$ be an interval such that $B G(u, v)$ is isomorphic to $B_{4}$, see Figure 1 . By Remark 10, we can label the first edges $u \rightarrow u_{1}, u \rightarrow u_{2}, u \rightarrow u_{3}, u \rightarrow u_{4}$ of $[u, v]$ by $(3,0),(2,1),(1,2),(0,3)$ respectively. The first edges $u_{1} \rightarrow u_{5}, u_{1} \rightarrow u_{6}, u_{1} \rightarrow u_{7}$ of $\left[u_{1}, v\right]$ are labeled by $(2,0),(1,1),(0,2)$ respectively. The images of the flip $\mathcal{F}$ on some paths in $B(u, v)$ are listed below.

$$
\begin{aligned}
& \mathcal{F}:\left(u \rightarrow u_{1} \rightarrow u_{5} \rightarrow v_{1} \rightarrow v\right) \mapsto\left(u \rightarrow u_{4} \rightarrow u_{10} \rightarrow v_{4} \rightarrow v\right), \\
& \mathcal{F}:\left(u \rightarrow u_{2} \rightarrow u_{5} \rightarrow v_{1} \rightarrow v\right) \mapsto\left(u \rightarrow u_{2} \rightarrow u_{9} \rightarrow v_{4} \rightarrow v\right), \\
& \mathcal{F}:\left(u \rightarrow u_{2} \rightarrow u_{8} \rightarrow v_{1} \rightarrow v\right) \mapsto\left(u \rightarrow u_{3} \rightarrow u_{8} \rightarrow v_{4} \rightarrow v\right), \\
& \mathcal{F}:\left(u \rightarrow u_{3} \rightarrow u_{6} \rightarrow v_{3} \rightarrow v\right) \mapsto\left(u \rightarrow u_{3} \rightarrow u_{10} \rightarrow v_{3} \rightarrow v\right) .
\end{aligned}
$$




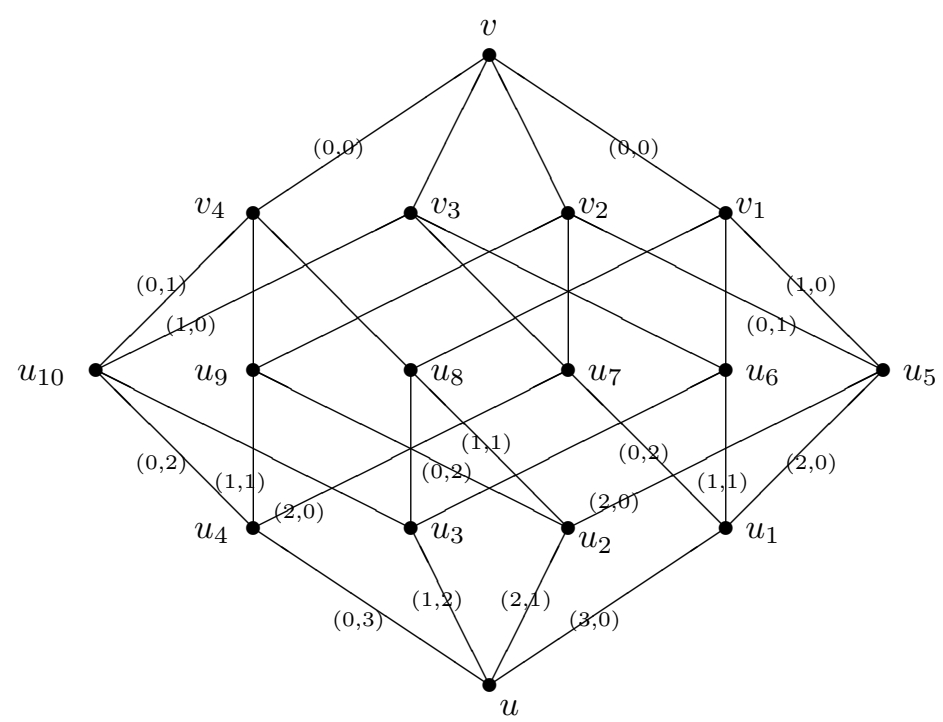

Figure 1: The Boolean lattice $B_{4}$.

Theorem 14. The flip $\mathcal{F}$ is compatible with the reflection order $\mathcal{O}$ and also satisfies the flip condition for any cd-monomial $M$.

Proof. We first show that the flip $\mathcal{F}$ satisfies the flip condition for any cd-monomial $M$. We claim that $s_{m, \mathbf{b}}(x) \neq-1$ for any $m$, any $\mathbf{c d - m o n o m i a l ~} M$ and any path $x$ in $B(u, v)$. Suppose to the contrary that there exists a path $x$ in $B(u, v)$ such that $s_{m, \mathbf{b}}(x)=-1$ for some integer $m$. Let

$$
x=\left(u \rightarrow x_{1} \rightarrow \cdots \rightarrow x_{m-1} \rightarrow x_{m} \rightarrow x_{m+1} \rightarrow x_{m+2} \rightarrow \cdots \rightarrow v\right) .
$$

Since each $\mathbf{b}$ follows by an $\mathbf{a}$ in $M(\mathbf{a}, \mathbf{b a})$, by the definitions of $s_{m, \mathbf{b}}(x)$ and $s_{m, \mathbf{a}}(x)$, we can assume that $x_{m-1}^{-1} x_{m} \prec x_{m}^{-1} x_{m+1} \prec x_{m+1}^{-1} x_{m+2}$. For $k=m, m+1, m+2$, let the label of the edge $x_{k-1} \rightarrow x_{k}$ be $\left(i_{k}, j_{k}\right)$. Then by Proposition 12, we have $i_{m}>i_{m+1}>i_{m+2}$.

To calculate $s_{m, \mathbf{b}}(x)$, we need to flip the path $x$ at $x_{m}$. Let

$$
y=\mathcal{F}_{x_{m}, v}(x)=\left(u \rightarrow x_{1} \rightarrow \cdots \rightarrow x_{m-1} \rightarrow x_{m} \rightarrow y_{m+1} \rightarrow y_{m+2} \rightarrow \cdots \rightarrow v\right) .
$$

If $x_{m+1}=y_{m+1}$ then we have $s_{m, \mathbf{b}}(x)=0$, a contradiction. If $x_{m+1} \neq y_{m+1}$, then by the definition of the flip $\mathcal{F}$, we find that $\min \left\{i_{m+1}, j_{m+1}\right\} \leqslant \min \left\{i_{m+2}, j_{m+2}\right\}$. Since $i_{m+1}>i_{m+2}$, we have $j_{m+1} \leqslant i_{m+2}$ and the label of the edge $x_{m} \rightarrow y_{m+1}$ must be $\left(j_{m+1}, i_{m+1}\right)$. Then we obtain $i_{m}>i_{m+1}>i_{m+2} \geqslant j_{m+1}$. Thus by Proposition 12 , $x_{m-1}^{-1} x_{m} \prec x_{m}^{-1} y_{m+1}$, and so $s_{m, \mathbf{b}}(x)=0$ again, which is a contradiction. This means $s_{m, \mathbf{b}}(x) \neq-1$ for any $m$. Thus the flip $\mathcal{F}$ satisfies the flip condition.

Now we proceed to show that the flip $\mathcal{F}$ is compatible with the reflection order $\mathcal{O}$. Let $M(\mathbf{c}, \mathbf{d})$ be a $\mathbf{c d}$-monomial with $M(\mathbf{a}, \mathbf{b a})=\gamma_{1} \cdots \gamma_{n-1}$. It suffices to show that for any integer $m \in[n-1]$ and any path $p$ in $B(u, v)$, we have $s_{m, \gamma_{m}}(p)=\bar{s}_{m, \gamma_{m}}(\mathcal{F}(p))$.

Assume that

$$
p=\left(u \rightarrow p_{1} \rightarrow \cdots \rightarrow p_{m-1} \rightarrow p_{m} \rightarrow p_{m+1} \rightarrow \cdots \rightarrow p_{n-1} \rightarrow v\right)
$$


is a path in $B(u, v)$ with label sequence

$$
\left(\left(r_{1}, s_{1}\right), \ldots,\left(r_{m}, s_{m}\right),\left(r_{m+1}, s_{m+1}\right), \ldots,\left(r_{n}, s_{n}\right)\right)
$$

in the reflection order $\mathcal{O}$. And let

$$
\mathcal{F}(p)=\left(u \rightarrow q_{1} \rightarrow \cdots \rightarrow q_{m-1} \rightarrow q_{m} \rightarrow q_{m+1} \rightarrow \cdots \rightarrow q_{n-1} \rightarrow v\right) .
$$

By the definition of the flip $\mathcal{F}$, the label sequence of $\mathcal{F}(p)$ is

$$
\begin{cases}\left(\left(r_{1}, s_{1}\right),\left(s_{2}, r_{2}\right), \ldots,\left(s_{m}, r_{m}\right),\left(s_{m+1}, r_{m+1}\right), \ldots,\left(s_{n}, r_{n}\right)\right), & \text { if } p_{1}=q_{1} \\ \left(\left(s_{1}, r_{1}\right),\left(s_{2}, r_{2}\right), \ldots,\left(s_{m}, r_{m}\right),\left(s_{m+1}, r_{m+1}\right), \ldots,\left(s_{n}, r_{n}\right)\right), & \text { if } p_{1} \neq q_{1} .\end{cases}
$$

It is easy to check that if $\gamma_{m}=\mathbf{a}$, then $s_{m, \mathbf{a}}(p)=\bar{s}_{m, \mathbf{a}}(\mathcal{F}(p))$. Now we consider the case $\gamma_{m}=\mathbf{b}$. To compute $s_{m, \mathbf{b}}(p)$, we need to flip the path $p$ at $p_{m}$. Let

$$
p^{\prime}=\mathcal{F}_{p_{m}, v}(p)=\left(u \rightarrow p_{1} \rightarrow \cdots \rightarrow p_{m-1} \rightarrow p_{m} \rightarrow p_{m+1}^{\prime} \rightarrow \cdots \rightarrow p_{n-1}^{\prime} \rightarrow v\right) .
$$

Then the label sequence of $p^{\prime}$ is

$$
\begin{cases}\left(\left(r_{1}, s_{1}\right), \ldots,\left(r_{m}, s_{m}\right),\left(r_{m+1}, s_{m+1}\right),\left(s_{m+2}, r_{m+2}\right), \ldots,\left(s_{n}, r_{n}\right)\right), & \text { if } p_{m+1}=p_{m+1}^{\prime} \\ \left(\left(r_{1}, s_{1}\right), \ldots,\left(r_{m}, s_{m}\right),\left(s_{m+1}, r_{m+1}\right),\left(s_{m+2}, r_{m+2}\right), \ldots,\left(s_{n}, r_{n}\right)\right), & \text { if } p_{m+1} \neq p_{m+1}^{\prime}\end{cases}
$$

To calculate $\bar{s}_{m, \mathbf{b}}(\mathcal{F}(p))$, we need to flip $\mathcal{F}(p)$ at $q_{m}$. Let

$$
\mathcal{F}_{q_{m}, v}(\mathcal{F}(p))=\left(u \rightarrow q_{1} \rightarrow \cdots \rightarrow q_{m-1} \rightarrow q_{m} \rightarrow q_{m+1}^{\prime} \rightarrow \cdots \rightarrow q_{n-1}^{\prime} \rightarrow v\right) .
$$

Then the label sequence of $\mathcal{F}_{q_{m}, v}(\mathcal{F}(p))$ is

$$
\begin{cases}\left(\left(r_{1}, s_{1}\right),\left(s_{2}, r_{2}\right), \ldots,\left(s_{m}, r_{m}\right),\left(s_{m+1}, r_{m+1}\right), \ldots,\left(r_{n}, s_{n}\right)\right), & \text { if } p_{1}=q_{1}, p_{m+1}=p_{m+1}^{\prime} \\ \left(\left(r_{1}, s_{1}\right),\left(s_{2}, r_{2}\right), \ldots,\left(s_{m}, r_{m}\right),\left(r_{m+1}, s_{m+1}\right), \ldots,\left(r_{n}, s_{n}\right)\right), & \text { if } p_{1}=q_{1}, p_{m+1} \neq p_{m+1}^{\prime} \\ \left(\left(s_{1}, r_{1}\right),\left(s_{2}, r_{2}\right), \ldots,\left(s_{m}, r_{m}\right),\left(s_{m+1}, r_{m+1}\right), \ldots,\left(r_{n}, s_{n}\right)\right), & \text { if } p_{1} \neq q_{1}, p_{m+1}=p_{m+1}^{\prime} \\ \left(\left(s_{1}, r_{1}\right),\left(s_{2}, r_{2}\right), \ldots,\left(s_{m}, r_{m}\right),\left(r_{m+1}, s_{m+1}\right), \ldots,\left(r_{n}, s_{n}\right)\right), & \text { if } p_{1} \neq q_{1}, p_{m+1} \neq p_{m+1}^{\prime}\end{cases}
$$

It is not hard to check that the paths $\mathcal{F}\left(p^{\prime}\right)$ and $\mathcal{F}_{q_{m}, v}(\mathcal{F}(p))$ have the same label sequence. Therefore,

$$
\mathcal{F}\left(p^{\prime}\right)=\mathcal{F}_{q_{m}, v}(\mathcal{F}(p)) .
$$

That is, if we flip the path $p$ at $p_{m}$ to obtain $p^{\prime}$, then under the flip $\mathcal{F}$, we shall flip $\mathcal{F}(p)$ at $q_{m}$ to obtain $\mathcal{F}\left(p^{\prime}\right)$.

Now we are prepared to show that $s_{m, \mathbf{b}}(p)=\bar{s}_{m, \mathbf{b}}(\mathcal{F}(p))$. If $m=1$ and $p_{1}=q_{1}$, then $p^{\prime}=\mathcal{F}(p)$ and $p=\mathcal{F}\left(p^{\prime}\right)$. It is obvious that $s_{1, \mathbf{b}}(p)=\bar{s}_{1, \mathbf{b}}(\mathcal{F}(p))$. If $m=1$ and $p_{1} \neq q_{1}$ or $m>1$, according to the label sequences of the paths $p, p^{\prime}, \mathcal{F}(p)$ and $\mathcal{F}\left(p^{\prime}\right)$ one can check that: (i) If $p_{m+1}=p_{m+1}^{\prime}$, then $s_{m, \mathbf{b}}(p)=\bar{s}_{m, \mathbf{b}}(\mathcal{F}(p))=0$. (ii) If $p_{m+1} \neq p_{m+1}^{\prime}$, then we also have $s_{m, \mathbf{b}}(p)=\bar{s}_{m, \mathbf{b}}(\mathcal{F}(p))$. This completes the proof. 
We are ready to give a combinatorial interpretation for the coefficients of $\widetilde{\psi}_{u, v}(\mathbf{c}, \mathbf{d})$.

Theorem 15. Let $M$ be a cd-monomial of degree $n-1$ such that $M(\mathbf{a}, \mathbf{b a})=\gamma_{1} \cdots \gamma_{n-1}$. Then the coefficient of $M$ in $\widetilde{\psi}_{u, v}(\mathbf{c}, \mathbf{d})$ is equal to the number of sequences

$$
\left(\left(i_{1}, j_{1}\right),\left(i_{2}, j_{2}\right), \ldots,\left(i_{n}, j_{n}\right)\right)
$$

satisfying the following conditions:

(1) For $1 \leqslant m \leqslant n, i_{m}, j_{m} \geqslant 0$ and $i_{m}+j_{m}=n-m$.

(2) For $1 \leqslant m \leqslant n-1$, if $\gamma_{m}=\mathbf{a}$ then $i_{m}>i_{m+1}$.

(3) For $1 \leqslant m \leqslant n-2$, if $\gamma_{m}=\mathbf{b}$ then $i_{m}, j_{m}>j_{m+1}$ and $i_{m+2} \geqslant j_{m+1}$.

Proof. According to Theorem 5, Corollary 8 and Theorem 14, the coefficient of $M$ is equal to the number of paths $x$ in $B(u, v)$ such that $s_{M}(x)=1$. Since a path is uniquely determined by its label sequence, we turn to the characterization of the corresponding sequences of nonnegative integer pairs $\left(\left(i_{1}, j_{1}\right),\left(i_{2}, j_{2}\right), \ldots,\left(i_{n}, j_{n}\right)\right)$ with $i_{k}+j_{k}=n-k$ for $1 \leqslant k \leqslant n$.

Let $x$ be the path in (2) with the edge $x_{m-1} \rightarrow x_{m}$ labeled by $\left(i_{m}, j_{m}\right)$ for $1 \leqslant m \leqslant n$. If $\gamma_{m}=\mathbf{a}$, then $s_{m, \mathbf{a}}(x)=1$ only if $x_{m-1}^{-1} x_{m} \prec x_{m}^{-1} x_{m+1}$. By Proposition 12, we have $i_{m}>i_{m+1}$. If $\gamma_{m}=\mathbf{b}$, then $\gamma_{m+1}=\mathbf{a}$. By Proposition 12, we have $i_{m+1}>i_{m+2}$. Since $i_{m+1}+j_{m+1}=i_{m+2}+j_{m+2}+1$, we see that $j_{m+1} \leqslant j_{m+2}$. After applying $\mathcal{F}_{x_{m}, v}$ to $x$, we get the path $y$ as in (3). We see that $s_{m, \mathbf{b}}(x)=1$ only if

$$
x_{m-1}^{-1} x_{m} \succ x_{m}^{-1} x_{m+1}, \quad x_{m-1}^{-1} x_{m} \prec x_{m}^{-1} y_{m+1} \quad \text { and } \quad x_{m+1} \neq y_{m+1} .
$$

Then the label of the edge $x_{m} \rightarrow y_{m+1}$ is $\left(j_{m+1}, i_{m+1}\right)$. By Proposition 12 and the definition of the flip $\mathcal{F}$, we find

$$
i_{m}, j_{m}>j_{m+1} \text { and } \min \left\{i_{m+1}, j_{m+1}\right\} \leqslant \min \left\{i_{m+2}, j_{m+2}\right\} .
$$

Combining with the facts $i_{m+1}>i_{m+2}$ and $j_{m+1} \leqslant j_{m+2}$, we obtain

$$
i_{m}, j_{m}>j_{m+1} \text { and } i_{m+1}>i_{m+2} \geqslant j_{m+1} \text {. }
$$

This completes the proof.

For example, let $[u, v]$ be a Bruhat interval such that $B G(u, v)$ is isomorphic to $B_{5}$. And let $M=\mathbf{d}^{2}$ with $M(\mathbf{a}, \mathbf{b a})=\mathbf{b a b a}$. There are 4 sequences corresponding to $M$ that satisfy the conditions in Theorem 15, namely,

$$
\begin{aligned}
& ((1,3),(3,0),(1,1),(1,0),(0,0)),((2,2),(2,1),(1,1),(1,0),(0,0)), \\
& ((2,2),(3,0),(1,1),(1,0),(0,0)),((3,1),(3,0),(1,1),(1,0),(0,0)) .
\end{aligned}
$$

Then the coefficient of $M$ in $\widetilde{\psi}_{u, v}(\mathbf{c}, \mathbf{d})$ is 4 . In fact,

$$
\widetilde{\psi}_{u, v}(\mathbf{c}, \mathbf{d})=\mathbf{c}^{4}+3 \mathbf{c}^{2} \mathbf{d}+5 \mathbf{c d c}+3 \mathbf{d} \mathbf{c}^{2}+4 \mathbf{d}^{2} .
$$




\begin{tabular}{|r|cllllll|c|}
\hline$m=$ & 1 & 2 & 3 & 4 & 5 & 6 & 7 & $E_{n}$ \\
\hline$n=1$ & 1 & & & & & & & 1 \\
2 & 1 & 0 & & & & & & 1 \\
3 & 1 & 1 & 0 & & & & & 2 \\
4 & 2 & 2 & 1 & 0 & & & & 5 \\
5 & 5 & 5 & 4 & 2 & 0 & & & 16 \\
6 & 16 & 16 & 14 & 10 & 5 & 0 & & 61 \\
7 & 61 & 61 & 56 & 46 & 32 & 16 & 0 & 272 \\
\hline
\end{tabular}

Table 1: The Entringer Numbers $E_{n}(k)$.

\section{Refined enumeration of $\widetilde{\psi}_{u, v}(1,1)$}

In this section, we give a refined enumeration of the number of $\mathbf{c d}$-monomials in $\widetilde{\psi}_{u, v}(\mathbf{c}, \mathbf{d})$ in terms of Entringer numbers.

Definition 16. A path $x$ in $B(u, v)$ is said to be valid if there exists some cd-monomial $M$ such that $s_{M}(x)=1$. A sequence $s=\left(\left(i_{1}, j_{1}\right),\left(i_{2}, j_{2}\right), \ldots,\left(i_{n}, j_{n}\right)\right)$ with $i_{k}, j_{k} \geqslant 0$ and $i_{k}+j_{k}=n-k(1 \leqslant k \leqslant n)$ is said to be valid if it corresponds to a valid path in $B(u, v)$. Equivalently, the sequence $s$ is said to be valid if $s$ satisfies: (i) $i_{n-1}>i_{n}$ and $i_{k}+j_{k}=n-k$ for $1 \leqslant k \leqslant n$; (ii) For $1 \leqslant k \leqslant n-2$, if $j_{k}>j_{k+1}$ then $i_{k}>j_{k+1}$ and $i_{k+1}>i_{k+2} \geqslant j_{k+1}$.

Now we enumerate the valid paths beginning with the same first edge. Let $E_{n}$ be the Euler number, i.e., the number of up-down permutations on $[n]$. It is well known that

$$
\tan u+\sec u=\sum_{n \geqslant 0} E_{n} \frac{u^{n}}{n !} .
$$

Denote by $E_{n}(k)$ the number of up-down permutations on $[n]$ beginning with $k(1 \leqslant k \leqslant$ $n)$. It is clear that $E_{n}=\sum_{k=1}^{n} E_{n}(k)$. The numbers $E_{n}(k)$ are called Euler and Bernoulli numbers, or Entringer numbers, see [6]. The Entringer numbers $E_{n}(k)$ for $n, k \leqslant 7$ are displayed in Table 1.

It is easy to verify that $E_{n}(n)=0$ and

$$
E_{n}(k)=\sum_{i=1}^{n-k} E_{n-1}(i), 1 \leqslant k \leqslant n-1 .
$$

Theorem 17. Suppose that the atoms of $[u, v]$ are $u_{1}, u_{2}, \ldots, u_{n}$, and the edge $u \rightarrow u_{k}$ has label $(n-k, k-1)$ for $1 \leqslant k \leqslant n$. Then the number of valid paths in $B(u, v)$ with first edge $u \rightarrow u_{k}$ is the Entringer number $E_{n}(k)$. In other words, the number of valid sequences beginning with $(n-k, k-1)$ is $E_{n}(k)$.

Proof. Let $T_{n}(k)$ denote the number of valid paths in $B(u, v)$ with first edge $u \rightarrow u_{k}$ labeled by $(n-k, k-1)$ for $1 \leqslant k \leqslant n$. It is easy to check that $T_{2}(1)=1, T_{2}(2)=0$. Then 
it suffices to show that $T_{n}(k)$ satisfies the relation (5). We analyze when a valid path in $B\left(u_{k}, v\right)$ can be extended to a valid path in $B(u, v)$.

For $1 \leqslant k \leqslant n$, let

$$
x^{\prime}=\left(u_{k} \rightarrow u_{k}^{i} \rightarrow u_{k}^{i j} \rightarrow \cdots \rightarrow v\right)
$$

be a valid path in $B\left(u_{k}, v\right)$. By Proposition 9 , we can label the edges $u_{k} \rightarrow u_{k}^{i}(1 \leqslant i \leqslant$ $n-1)$ and $u_{k}^{i} \rightarrow u_{k}^{i j}(1 \leqslant j \leqslant n-2)$ by $(n-i-1, i-1)$ and $(n-j-2, j-1)$ respectively. By induction, $T_{n-1}(t)$ is the number of valid paths in $B\left(u_{k}, v\right)$ beginning with $u_{k} \rightarrow u_{k}^{t}$ for $1 \leqslant t \leqslant n-1$. Moreover, $T_{n-1}(n-1)=0$ and for $1 \leqslant r \leqslant n-2$,

$$
T_{n-1}(r)=\sum_{i=1}^{n-r-1} T_{n-2}(i) .
$$

Now we extend the path $x^{\prime}$ to a path $x$ in $B(u, v)$. Let

$$
x=\left(u \rightarrow u_{k} \rightarrow u_{k}^{i} \rightarrow u_{k}^{i j} \rightarrow \cdots \rightarrow v\right) .
$$

Since $x^{\prime}$ is a valid path in $B\left(u_{k}, v\right), x$ is a valid path in $B(u, v)$ if and only if $s_{1, \mathbf{a}}(x)=1$ or $s_{1, \mathbf{b}}(x)=1$. To compute $s_{1, \mathbf{a}}(x)$ or $s_{1, \mathbf{b}}(x)$, we need to flip the path $x$ at $u_{k}$. Let

$$
y=\mathcal{F}_{u_{k}, v}(x)=\left(u \rightarrow u_{k} \rightarrow\left(u_{k}^{i}\right)^{\prime} \rightarrow\left(u_{k}^{i j}\right)^{\prime} \rightarrow \cdots \rightarrow v\right) .
$$

Then $x$ is a valid path in $B(u, v)$ if and only if $u^{-1} u_{k} \prec u_{k}^{-1} u_{k}^{i}$, or $u^{-1} u_{k} \succ u_{k}^{-1} u_{k}^{i}$ and $u^{-1} u_{k} \prec u_{k}^{-1}\left(u_{k}^{i}\right)^{\prime}$ and $u_{k}^{i} \neq\left(u_{k}^{i}\right)^{\prime}$. Since the edge $u \rightarrow u_{k}$ has label $(n-k, k-1)$, by (4), we deduce that $x$ is a valid path in $B(u, v)$ if and only if

$$
n-k>n-i-1
$$

or

$$
k-1>i-1, n-k>i-1 \text { and } n-i-1>n-j-2 \geqslant i-1 .
$$

If (6) holds, then we have $k \leqslant i \leqslant n-1$. Hence by induction, the number of valid paths in $B(u, v)$ extended from the first edges $u_{k} \rightarrow u_{k}^{n-1}, \ldots, u_{k} \rightarrow u_{k}^{k}$ in $B\left(u_{k}, v\right)$ is

$$
\sum_{i=k}^{n-1} T_{n-1}(i) \text {. }
$$

If (7) holds, then we get

$$
1 \leqslant i \leqslant \alpha \text { and } i \leqslant j \leqslant n-i-1 \text {, }
$$

where $\alpha=\min \{n-k, k-1\}$. Thus by induction, the number of valid paths in $B\left(u_{k}, v\right)$ which begin with $u_{k} \rightarrow u_{k}^{i}(1 \leqslant i \leqslant \alpha)$ and can be extended to a valid path in $B(u, v)$ is

$$
\begin{aligned}
T_{n-1}^{\prime}(i) & =\sum_{j=i}^{n-i-1} T_{n-2}(j) \\
& =T_{n-1}(1)-\sum_{r=1}^{i-1}\left(T_{n-2}(r)+T_{n-2}(n-r-1)\right) .
\end{aligned}
$$


Combining (8), (9) and by induction, we derive that

$$
\begin{aligned}
T_{n}(k) & =\sum_{i=1}^{\alpha} T_{n-1}^{\prime}(i)+\sum_{i=k}^{n-1} T_{n-1}(i) \\
& =\sum_{i=1}^{\alpha}\left(T_{n-1}(1)-\sum_{r=1}^{i-1}\left(T_{n-2}(r)+T_{n-2}(n-r-1)\right)\right)+\sum_{i=k}^{n-1} T_{n-1}(i) \\
& =\alpha T_{n-1}(1)-\sum_{i=1}^{\alpha} T_{n-1}(n-i)-\sum_{i=1}^{\alpha}\left(T_{n-1}(1)-T_{n-1}(i)\right)+\sum_{i=k}^{n-1} T_{n-1}(i) \\
& =\sum_{i=1}^{\alpha}\left(T_{n-1}(i)-T_{n-1}(n-i)\right)+\sum_{i=k}^{n-1} T_{n-1}(i) .
\end{aligned}
$$

Since $\alpha=n-k$ or $k-1$, and it is easy to check that

$$
\sum_{i=1}^{n-k}\left(T_{n-1}(i)-T_{n-1}(n-i)\right)=\sum_{i=1}^{k-1}\left(T_{n-1}(i)-T_{n-1}(n-i)\right),
$$

we find that

$$
\sum_{i=1}^{\alpha}\left(T_{n-1}(i)-T_{n-1}(n-i)\right)=\sum_{i=1}^{k-1}\left(T_{n-1}(i)-T_{n-1}(n-i)\right) .
$$

It follows from (10) and (11) that

$$
T_{n}(k)=\sum_{i=1}^{k-1}\left(T_{n-1}(i)-T_{n-1}(n-i)\right)+\sum_{i=k}^{n-1} T_{n-1}(i)=\sum_{i=1}^{n-k} T_{n-1}(i),
$$

as desired. This completes the proof.

Corollary 18. The number of valid paths in $B(u, v)$ or the number of valid sequences of length $n$ is equal to the Euler number $E_{n}$. In other words, $\widetilde{\psi}_{u, v}(1,1)=\psi_{B_{n}}(1,1)=E_{n}$.

It is worth mentioning that Billera [1] conjectured that for all lower Bruhat intervals $[e, v], \widetilde{\psi}_{e, v}(1,1) \leqslant \psi_{B_{\ell(v)}}(1,1)$. In our words, this conjecture asserts that for all lower Bruhat intervals $[e, v]$ such that $\ell(v)=n$, if there exists a flip on the set of paths in $B(e, v)$ satisfies the flip condition, then the number of valid paths in $B(e, v)$ is less than or equal to $E_{n}$.

\section{The coefficient of $\mathrm{d}^{n}$}

In this section, we interprete the coefficient of $\mathbf{d}^{n}$ in terms of the Poupard numbers. 


$$
\begin{array}{cccccccc} 
& & 0 & 1 & 0 & & & \\
& 0 & 1 & 2 & 1 & 0 & & \\
0 & 4 & 8 & 10 & 8 & 4 & 0 & \\
34 & 68 & 94 & 104 & 94 & 68 & 34 & 0
\end{array}
$$

Table 2: The Poupard triangle $P_{n}(k)$.

The Poupard numbers $P_{n}(k)(1 \leqslant k \leqslant 2 n+1)$ are defined recursively as follows, see Poupard [14] or Foata and Han [8]. Let $P_{1}(1)=0, P_{1}(2)=1, P_{1}(3)=0$, and $P_{n}(1)=0$, $P_{n}(2)=\sum_{j=1}^{2 n-1} P_{n-1}(j)$ for $n \geqslant 2$. For $3 \leqslant k \leqslant 2 n+1$,

$$
P_{n}(k)=2 P_{n}(k-1)-P_{n}(k-2)-2 P_{n-1}(k-2) .
$$

By [8, Corollary 4.3], we have

$$
P_{n}(k)=P_{n}(2 n-k+2) \text { for } 1 \leqslant k \leqslant n .
$$

The first few lines of the Poupard triangle are listed in Table 2.

Recall that the numbers $t_{n}$ appearing in the Taylor expansion

$$
\begin{aligned}
\sqrt{2} \tan (u / \sqrt{2}) & =\sum_{n \geqslant 0} t_{n} \frac{u^{2 n+1}}{(2 n+1) !} \\
& =1 \frac{u}{1 !}+1 \frac{u^{3}}{3 !}+4 \frac{u^{5}}{5 !}+34 \frac{u^{7}}{7 !}+496 \frac{u^{9}}{9 !}+11056 \frac{u^{11}}{11 !}+\cdots
\end{aligned}
$$

are called the reduced tangent numbers. It is easy to see that $t_{n}=E_{2 n+1} / 2^{n}$, where $E_{2 n+1}$ are the Euler numbers. By [8, Theorem 1.1], we have

$$
t_{n}=\sum_{k=1}^{2 n+1} P_{n}(k) .
$$

Definition 19. A valid path $x$ in $B(u, v)$ of length $2 n+1$ is said to be alternating if $w(x)=$ baba $\cdots$ ba. The label sequence $s=\left(\left(i_{1}, j_{1}\right),\left(i_{2}, j_{2}\right), \ldots,\left(i_{2 n+1}, j_{2 n+1}\right)\right)$ of an alternating path is called an alternating sequence. Equivalently, the sequence $s$ is said to be alternating if $s$ is valid and $j_{2 r-1}>j_{2 r}$ and $i_{2 r}>i_{2 r+1}$ for $1 \leqslant r \leqslant n$.

Theorem 20. Let $[u, v]$ be a Bruhat interval such that $B G(u, v)$ is isomorphic to the Boolean lattice $B_{2 n+1}$. Suppose that the atoms of $[u, v]$ are $u_{1}, u_{2}, \ldots, u_{2 n+1}$, and the edge $u \rightarrow u_{k}(1 \leqslant k \leqslant 2 n+1)$ has label $(2 n-k+1, k-1)$. Then the number of alternating paths in $B(u, v)$ beginning with the edge $u \rightarrow u_{k}$ is the Poupard number $P_{n}(k)$. In other words, the number of alternating sequences of length $2 n+1$ beginning with $(2 n-k+1, k-1)$ is $P_{n}(k)$. 
Proof. Assume that $F_{n}(k)$ is the number of alternating paths in $B(u, v)$ with first edge $u \rightarrow u_{k}$ labeled by $(2 n-k+1, k-1)$ for $1 \leqslant k \leqslant 2 n+1$. It is easy to check that $F_{1}(1)=0, F_{1}(2)=1, F_{1}(3)=0$. We claim that for $1 \leqslant k \leqslant n+1$,

$$
F_{n}(k)=\sum_{i=1}^{k-1} \sum_{j=i}^{2 n-i} F_{n-1}(j)
$$

and for $n+1<k \leqslant 2 n+1$,

$$
F_{n}(k)=F_{n}(2 n-k+2) .
$$

We prove (13) first. Let

$$
x=\left(u \rightarrow u_{k} \rightarrow u_{k}^{i} \rightarrow u_{k}^{i j} \rightarrow \cdots \rightarrow v\right)
$$

be a path in $B(u, v)$. By Proposition 9, we can assume that the edges $u \rightarrow u_{k}, u_{k} \rightarrow u_{k}^{i}$ and $u_{k}^{i} \rightarrow u_{k}^{i j}$ are labeled by $(2 n-k+1, k-1),(2 n-i, i-1)$ and $(2 n-j-1, j-1)$ respectively. Since the path $x$ is valid and $w(x)=\mathbf{b a b a} \cdots \mathbf{b a}$, by Theorem 15 , we see that

$$
k-1>i-1,2 n-k+1>i-1,
$$

and

$$
2 n-j-1 \geqslant i-1,2 n-i>2 n-j-1 .
$$

Suppose that

$$
x^{\prime \prime}=\left(u_{k}^{i} \rightarrow u_{k}^{i j} \rightarrow \cdots \rightarrow v\right)
$$

is an alternating path in $B\left(u_{k}^{i}, v\right)$. By induction, the number of alternating paths in $B\left(u_{k}^{i}, v\right)$ with first edge $u_{k}^{i} \rightarrow u_{k}^{i j}$ is $F_{n-1}(j)$.

If $1 \leqslant k \leqslant n+1$, then we have $2 n-k+1 \geqslant k-1$. By (15), we see that $i \leqslant k-1$. By (16), we have $i \leqslant j \leqslant 2 n-i$. That is to say, only the paths beginning with $u_{k} \rightarrow$ $u_{k}^{i}(1 \leqslant i \leqslant k-1)$ and $u_{k}^{i} \rightarrow u_{k}^{i j}(i \leqslant j \leqslant 2 n-i)$ will contribute to $F_{n}(k)$. Therefore, the equation (13) holds.

If $n+1<k \leqslant 2 n+1$, i.e., $2 n-k+1<k-1$, then by (15) we have $i \leqslant 2 n-k+1$ and by (16), we have $i \leqslant j \leqslant 2 n-i$. Let $k^{\prime}=2 n-k+2$, then $1 \leqslant k^{\prime} \leqslant n$ and so

$$
F_{n}(k)=\sum_{i=1}^{2 n-k+1} \sum_{j=i}^{2 n-i} F_{n-1}(j)=\sum_{i=1}^{k^{\prime}-1} \sum_{j=i}^{2 n-i} F_{n-1}(j)=F_{n}\left(k^{\prime}\right)=F_{n}(2 n-k+2) .
$$

Thus the equation (14) holds. 
Now we show that $F_{n}(k)(1 \leqslant k \leqslant n+1)$ satisfies the relation (12). By (13), we have

$$
\begin{aligned}
& 2 F_{n}(k-1)-F_{n}(k-2)-2 F_{n-1}(k-2) \\
& =2 \sum_{i=1}^{k-2} \sum_{j=i}^{2 n-i} F_{n-1}(j)-\sum_{i=1}^{k-3} \sum_{j=i}^{2 n-i} F_{n-1}(j)-2 F_{n-1}(k-2) \\
& =\sum_{i=1}^{k-2} \sum_{j=i}^{2 n-i} F_{n-1}(j)+\sum_{j=k-2}^{2 n-k+2} F_{n-1}(j)-2 F_{n-1}(k-2) \\
& =\sum_{i=1}^{k-2} \sum_{j=i}^{2 n-i} F_{n-1}(j)+\sum_{j=k-1}^{2 n-k+1} F_{n-1}(j) \\
& =\sum_{i=1}^{k-1} \sum_{j=i}^{2 n-i} F_{n-1}(j)=F_{n}(k),
\end{aligned}
$$

where the third equation follows from the fact $F_{n-1}(k-2)=F_{n-1}(2 n-k+2)$, which holds by induction. This completes the proof.

Corollary 21. The Poupard numbers $P_{n}(k)$ can be defined recursively as follows. For $1 \leqslant k \leqslant n+1$

$$
P_{n}(k)=\sum_{i=1}^{k-1} \sum_{j=i}^{2 n-i} P_{n-1}(j),
$$

where $P_{1}(1)=0, P_{1}(2)=1, P_{1}(3)=0$ and $P_{n}(1)=0$ for all $n \geqslant 1$, and $P_{n}(k)=$ $P_{n}(2 n-k+2)$ for $n+1<k \leqslant 2 n+1$.

The following corollary was also obtained by Mahajan [13] algebraically.

Corollary 22. The coefficient of $\mathbf{d}^{n}$ is the reduced tangent number $t_{n}$.

To conclude, we remark that since the cd-index of $B_{n}$ depends only on the poset structure of $B_{n}$ and $\widetilde{\psi}_{u, v}(\mathbf{c}, \mathbf{d})=\psi_{u, v}(\mathbf{c}, \mathbf{d})$ when $B G(u, v)$ is isomorphic to $B_{n}$, the complete cd-index of $[u, v]$ is combinatorial invariant. However, there lacks of a direct proof of this fact in the viewpoint of the complete cd-index. In this paper, we provide such a proof by labeling the edges of $[u, v]$ by pairs of nonnegative integers, and show that this labeling is independent of the specific interval $[u, v]$ as long as its Bruhat graph is isomorphic to $B_{n}$. Then we can compute the ab-polynomial $\widetilde{\phi}_{u, v}(\mathbf{a}, \mathbf{b})$ of $[u, v]$ according to this labeling (Proposition 12), and get rid of the specific Coxeter group.

It would be interesting to find a direct correspondence between the set of up-down permutations on $[n]$ beginning with $k(1 \leqslant k \leqslant n)$ and the set of valid sequences of length $n$ beginning with $(n-k, k-1)$. 


\section{References}

[1] L. J. Billera. Flag enumeration in polytopes, Eulerian partially ordered sets and Coxeter groups. Proceedings of the International Congress of Mathematicians, vol. IV, pages 2389-2415. Hindustan Book Agency, New Delhi, 2010.

[2] L. J. Billera and F. Brenti. Quasisymmetric functions and Kazhdan-Lusztig polynomials. Isr. J. Math., 184:317-348, 2011.

[3] A. Björner and F. Brenti. Combinatorics of Coxeter groups. Graduate Texts in Mathematics, Vol. 231, Springer-Verlag, New York, 2005.

[4] M. J. Dyer. Hecke algebras and shellings of Bruhat intervals. Compos. Math., 89:91$115,1993$.

[5] M. J. Dyer. Proof of Cellini's conjecture on self-avoiding paths in Coxeter groups. Compos. Math., 148:548-554, 2012.

[6] R. C. Entringer. A combinatorial interpretation of the Euler and Bernoulli numbers. Nieuw. Arch. Wisk., 14:241-246, 1966.

[7] N. J. Y. Fan and Liao He. On the non-negativity of the complete cd-index. Discrete Math., to appear.

[8] D. Foata and Guo-Niu Han. The doubloon polynomial triangle. Ramanujan J., 23:107-126, 2010.

[9] G. Hetyei. On the cd-variation polynomials of André and simsun permutations. Discrete Comput. Geom., 16(3):259-275, 1996.

[10] J. E. Humphreys. Reflection groups and Coxeter groups. Cambridge Studies in Advanced Mathematics, Cambridge Univ. Press, Cambridge, 1990.

[11] K. Karu. On the complete cd-index of a Bruhat interval. J. Algebraic Comb., 38:527$541,2013$.

[12] D. Kazhdan and G. Lusztig. Representations of Coxeter groups and Hecke algebras. Invent. Math., 53:165-184, 1979.

[13] S. Mahajan. The cd-index of the Boolean lattice. 2002. arXiv:math/0211390

[14] C. Poupard. Deux propriétés des arbres binaires ordonnés stricts. Eur. J. Comb., 10:369-374, 1989.

[15] M. Purtill. André permutations, lexicographic shellability and the cd-index of a convex polytope. Trans. Amer. Math. Soc., 338:77-104, 1993.

[16] R. P. Stanley. A survey of Eulerian posets. In "Polytopes: Abstract, Convex, and Computational", T. Bisztriczky, P. McMullen, R. Schneider, A. I. Weiss, eds., NATO ASI Series C, Vol. 440, Kluwer Academic Publishers, 1994.

[17] S. Sundaram. The homology representation of the symmetric group on CohenMacaulay subposets of the partition lattice. Adv. Math., 104:225-296, 1994.

[18] S. Sundaram. The homology of partitions with an even number of blocks. J. Algebraic Comb., 4:69-92, 1995. 